\title{
Poliartrite em cães - 27 casos (2007-2013)
}

\author{
Polyarthritis in dogs -27 cases (2007-2013)
}

\author{
Rafael Oliveira Chaves ${ }^{\mathrm{I}}$ Diego Vilibaldo Beckmann ${ }^{\mathrm{IV}}$ Bruna Copat $^{\mathrm{I}}$ \\ Raquel Baumhardt ${ }^{I}$ Amanda Oliveira Andrades ${ }^{I}$ Laís Barbieri Silveira ${ }^{I I}$ \\ Ana Paula da Silva ${ }^{I}$ Alexandre Mazzanti ${ }^{I I I}$
}

\section{RESUMO}

\begin{abstract}
O objetivo deste estudo foi identificar cães com poliartrite e obter informações a respeito da idade, sexo, raça, peso corporal, queixa principal, alterações no exame físico, do líquido sinovial e resposta à terapia instituída. Os cães sem raça definida e acima de seis anos de idade foram os mais acometidos e o principal sinal clínico observado foi dificuldade de locomoção $(71,5 \%)$, seguido de dor articular (52,3\%) e claudicação (33,3\%). Febre e inapetência foram observadas em cinco $(18,5 \%)$ e quatro cães (14,8\%), respectivamente. As principais alterações hematológicas encontradas foram anemia não regenerativa $(29,6 \%)$, leucocitose (26\%) e trombocitopenia (18,5\%). Os resultados da bioquímica sérica foram considerados normais em sete de 27 cães (26\%) $e$ as principais alterações foram hiperproteinemia (55,5\%) $e$ hipoalbuminemia (37\%). A inflamação sinovial foi considerada acentuada em 17 cães (77,5\%), sendo os neutrófilos predominantes em 52,4\% dos casos $(n=14)$. O tratamento foi realizado em 18 cães com doses imunossupressoras de corticosteroides, sendo efetivo em $89 \%$ dos casos.
\end{abstract}

Palavras-chave: articulação, líquido sinovial, cães.

\section{ABSTRACT}

The aim of this study was to identify dogs with polyarthritis (PA) and information about age, sex, race, body weight, chief complaint, changes in physical examination, cytology of synovial fluid and response to therapy. Mixed breeds dogs over six years of age were the most affected and the main symptoms observed were limited mobility (71.5\%) followed by joint pain $(52.3 \%)$ and claudication (33.3\%). Inapetence and fever were observed in five (18.5\%) and four $\operatorname{dogs}(14.8 \%)$, respectively. Haematological abnormalities as non regenerative anemia
(29.6\%), leukocytosis (26\%) and thrombocytopenia (18.5\%) were found. The results of serum biochemistry were normal in seven of 27 dogs (26\%) and the major changes were hyperproteinemia (55.5\%) and hypoalbuminemia (37\%). The synovial inflammation was considered severe in 17 dogs (77.5\%) and neutrophils prevalent in $52.4 \%$ of cases $(n=14)$. The treatment was performed in 18 dogs with immunosuppressive doses of corticosteroids, and was effective in $89 \%$ of cases.

Key words: joints, synovial fluid, dogs.

\section{INTRODUÇÃO}

Poliartrite é uma artropatia inflamatória que acomete mais de uma articulação (JACQUES et al., 2002; GOLDSTEIN, 2010; JOHNSON \& MACKIN, 2012a). Em cães, essa afecção é considerada pouco frequente e quase sempre de origem infecciosa ou imunomediada e raramente induzida por cristais (JACQUES et al., 2002; RONDEAU et al., 2005; GOLDSTEIN, 2010; WILSON-WAMBOLDT, 2011; JOHNSON \& MACKIN, 2012a). Poliartrites infecciosas podem ser causadas por vírus, bactérias, protozoários (SPRENG, 1993; MCCONKEY et al., 2002; SANTOS et al., 2006), ou fungos (RONDEAU et al., 2005; GOLDSTEIN, 2010; WILSONWAMBOLDT, 2011). As artrites induzidas por

IPrograma de Pós-graduação em Medicina Veterinária, Centro de Ciências Rurais (CCR), Universidade Federal de Santa Maria (UFSM), Camobi, Santa Maria, RS, Brasil.

${ }^{\text {IIC }}$ urso de Medicina Veterinária, CCR, UFSM, Santa Maria, RS, Brasil.

IIIDepartamento de Clínica de Pequenos Animais, CCR, UFSM, 97105-900, Santa Maria, RS, Brasil. E-mail: alexamazza@yahoo.com.br. Autor para correspondência.

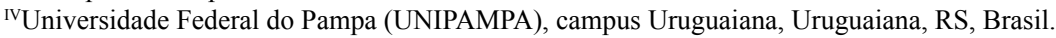


cristal são ocasionadas pela deposição de cristais de urato de sódio (gota) ou de pirofosfato de cálcio di-hidratado (pseudogota) (THOREN-TOLLING \& RYDEN, 1991; DE HAAN \& ANDREASEN, 1992; HOPPER, 1993; GOLDSTEIN, 2010) e as de etiologia imunomediada são decorrentes de um estímulo imunológico que desencadeia a formação e a deposição de imunocomplexos e liberação de enzimas lisossomais no interior da membrana sinovial (hipersensibilidade do tipo III) (HOPPER, 1993; JACQUES et al., 2002; GOLDSTEIN, 2010).

Os sinais clínicos da poliartrite são variáveis e incluem dificuldade na locomoção, rigidez articular, diminuição da amplitude de movimento, claudicação de um ou mais membros, que pode ser intermitente, vocalização, incapacidade de ficar em pé ou caminhar, derrame articular e artralgia. Às vezes, sinais sistêmicos, como febre, anorexia, inapetência e perda de peso podem ser os únicos observados (GOLDSTEIN, 2010; WILSONWAMBOLDT, 2011; JOHNSON \& MACKIN, 2012b). As articulações mais afetadas são carpo, tarso, joelho e cotovelo (CLEMENTS et al., 2004).

$\mathrm{O}$ diagnóstico da poliartrite se baseia no exame clínico, com ênfase no exame ortopédico, na avaliação laboratorial pelo aumento de leucócitos no líquido sinovial de diversas articulações. Vários protocolos de tratamento têm sido propostos e pode envolver um único fármaco ou uma combinação de medicamentos, incluindo corticosteroides e agentes citotóxicos ou imunomoduladores. A melhor forma de avaliar a eficácia terapêutica é mediante a remissão dos sinais clínicos e principalmente pela avaliação citológica do líquido sinovial (COLOPY et al., 2010; JOHNSON \& MACKIN, 2012b).

Alguns estudos sobre poliartrite são encontrados na literatura, basicamente internacional (JACQUES et al., 2002; WEBB et al., 2002; CLEMENTS et al., 2004; RONDEAU et al., 2005; STULL et al., 2008), e na forma de relatos de casos (THILAGAR et al., 1990; WOODARD et al., 1991; SPRENG, 1993; MCCONKEY et al., 2002; SANTOS et al., 2006; WILSON-WAMBOLDT, 2011). Na literatura nacional, no entanto, poucos dados e estudos retrospectivos são obtidos sobre o assunto.

Diante disso, o objetivo deste trabalho foi identificar cães com poliartrite, atendidos em um Serviço de Neurologia de um hospital veterinário e obter informações a respeito da idade, sexo, raça, peso corporal, queixa principal, alterações no exame físico, avaliação laboratorial do líquido sinovial e resposta à terapia instituída.

\section{MATERIAL E MÉTODOS}

Foram revisados os arquivos do Serviço de Neurologia do Hospital Veterinário Universitário (HVU) da Universidade Federal de Santa Maria (UFSM) no período entre janeiro de 2007 e março de 2013. Foram incluídos, nesse estudo, cães que apresentavam dados completos de história clínica, sinais clínicos, exame clínico e neurológico, com evidências citológicas de inflamação confirmada através da análise do líquido sinovial de duas ou mais articulações.

Foram avaliados idade, sexo, raça, peso corporal, queixa principal, alterações no exame físico, resultado citológico do líquido sinovial e resposta à terapia. Em todos os casos, foram realizados métodos complementares de diagnóstico, como hemograma, bioquímica sérica (ureia, creatinina, glicose, alanina aminotransferase (ALT), fosfatase alcalina (FA), proteína total, albumina e cálcio) e urinálise.

O líquido sinovial foi obtido através de artrocentese de pelo menos duas articulações (geralmente, carpo e joelho) em cada cão sob sedação ou anestesia geral. A punção da articulção foi realizada com agulha $25 \times 8$ e o material aspirado com uma serginga de $10 \mathrm{ml}$, precedida de antissepsia local com álcool-iodo-álcool. As análises foram realizadas no Laboratório de Análises Clínicas da mesma instituição. $\mathrm{Na}$ avaliação física, as amostras foram caracterizadas apenas quanto à coloração, no exame químico, as amostras foram avaliadas atráves da quantificação de proteína total (BOON, 1997; GIBSON et al., 1999; MACWILLIAMS \& FRIEDRICHS, 2003). A avaliação citológica do líquido sinovial foi realizada através da análise de esfregaços corados com WrightGiemsa. O diferencial leucocitário foi realizado através da observação de 10 campos de células. O líquido sinovial foi considerado normal se menos de duas células/campo fossem encontradas em um aumento de 400 vezes e seu diferencial apresentasse menos de $12 \%$ de neutrófilos (BOON, 1997; GIBSON et al., 1999; MACWILLIAMS \& FRIEDRICHS, 2003).

A contagem celular média dos líquidos sinoviais das articulações foi calculada e caracterizada em diferentes graus de inflamação e classificado como leve ( 2 a 10 células campo ${ }^{-1}$ de $\left.400 \mathrm{X}\right)$, moderado (10 a 50 células) e acentuado ( $>50$ células).

Radiografias (projeções dorsoventral e lateral) foram realizadas com base nos resultados da análise do líquido sinovial ou caso o paciente apresentasse aumento de volume na articulação, para exclusão de artrite erosiva.

O protocolo terapêutico instituído foi com prednisolona na dose de $4 \mathrm{mg} \mathrm{kg}^{-1}$, via oral, a cada 
24 horas, durante quatro dias. Após, $3 \mathrm{mg} \mathrm{kg}^{-1}$, a cada 24 horas, durante três dias. No oitavo dia, a dose foi reduzida para $2 \mathrm{mg} \mathrm{kg}^{-1}$, a cada 24 horas, por 15 dias, seguido de $1 \mathrm{mg} \mathrm{kg}^{-1}$, a cada 24 horas, por mais 21 dias. Em seguida, foi administrado $0,5 \mathrm{mg} \mathrm{kg}^{-1}$, a cada 24 horas, por mais sete dias e, a cada 48 horas, por mais sete dias. Decorridos cinquenta e sete dias de tratamento, foram realizados exames do líquido sinovial, conforme técnica descrita anteriormente.

A análise estatística dos dados obtidos foi pelo teste do qui-quadrado, a 5\% de significância.

\section{RESULTADOS E DISCUSSÃO}

Entre 2007 e 2013, foram atendidos 1128 cães pelo Serviço de Neurologia do HVU-UFSM, desses, 27 (2,4\%) cães foram incluídos de acordo com os critérios pré-estabelecidos. A distribuição etária variou de sete meses a treze anos, com uma média de seis anos e seis meses, sendo 55,5\% fêmeas (15 de 27 cães) e 44,5\% machos (12 de 27 cães). Os cães sem raça definida foram os mais acometidos (26\%), seguidos pelo dachshund $(11,1 \%)$, labrador $(7,4 \%)$, cocker $(7,4 \%)$, collie $(7,4 \%)$, poodle $(7,4 \%)$, rottweiler $(7,4 \%)$, boxer $(3,7 \%)$, maltês $(3,7 \%)$, pointer $(3,7 \%)$, chow chow $(3,7 \%)$, samoieda $(3,7 \%)$, border collie $(3,7 \%)$ e lhasa apso $(3,7 \%)$ (Tabela 1$)$. Em outros estudos retrospectivos, como o de JACQUES et al. (2002), CLEMENTS et al. (2004), STULL et al. (2008) e COLOPY et al. (2010), foi descrita predisposição de determinadas raças ou sexo em relação à poliartrite imunomediada, porém não há consenso entre esses trabalhos. Quanto ao peso corporal, a média foi de $19,5 \mathrm{~kg}$ (faixa 3,9 a

Tabela 1 - Representação quanto à raça, sexo, idade, grau de inflamação citológica, principal sinal clínico e tempo de recuperação de 27 cães com poliartrite, atendidos HVU-UFSM no período entre 2007 e 2013.

\begin{tabular}{|c|c|c|c|c|}
\hline $\begin{array}{l}\text { Raça/ano de } \\
\text { atendimento/Sexo }\end{array}$ & Idade & Principal sinal clínico & $\begin{array}{l}\text { Grau de inflamação } \\
\text { citológica }\end{array}$ & $\begin{array}{l}\text { Tempo de recuperação } \\
\text { (dias) }\end{array}$ \\
\hline $\mathrm{SRD} / 2007 / \mathrm{M}$ & 7 anos & Dificuldade de locomoção e claudicação & Leve & SI \\
\hline Cocker/2009/M & 11 anos & Claudicação & Acentuado & 60 \\
\hline Collie/2009/M & 11 meses & Dificuldade de locomoção e andar rígido & Acentuado & 30 \\
\hline Cocker/2010/M & 6 anos & Dificuldade de locomoção e hiporexia & Acentuado & $30 *$ \\
\hline Boxer/2011/F & 2 anos & Dificuldade de locomoção & Acentuado & SI \\
\hline $\mathrm{SRD} / 2011 / \mathrm{F}$ & 11 anos & Dificuldade de locomoção & Acentuado & SI \\
\hline Labrador/2011/M & 3 anos & Claudicação & Acentuado & $\mathrm{SM}^{* *}$ \\
\hline Dachshund/2011/M & 9 anos & Dificuldade de locomoção e dor & Acentuado & $7 * * *$ \\
\hline Collie/2011/F & 7 meses & Dificuldade de locomoção & Acentuado & 15 \\
\hline $\mathrm{SRD} / 2011 / \mathrm{M}$ & 2 anos & Dificuldade de locomoção e andar rígido & Acentuado & 30 \\
\hline Dachshund/2011/F & 7 anos & Dificuldade de locomoção & Acentuado & 20 \\
\hline Poodle/2011/F & 10 anos & $\begin{array}{l}\text { Dificuldade de locomoção e aumento de } \\
\text { volume nas articulações }\end{array}$ & Acentuado & SI \\
\hline Lhasa apso/2011/F & 4 anos & Dificuldade de locomoção & Sem contagem & SI \\
\hline Rottweiler/2012/M & 3 anos & Dificuldade de locomoção e fraqueza & Acentuado & SI \\
\hline Pointer/2012/F & 8 anos & Claudicação & Acentuado & SI \\
\hline Chow chow/2012/F & 7 anos & Dificuldade de locomoção & Acentuado & 20 \\
\hline Labrador/2012/M & 12 anos & Claudicação & Acentuado & $7 * * * *$ \\
\hline $\mathrm{SRD} / 2012 / \mathrm{F}$ & 13 anos & Dificuldade de locomoção e andar rígido & Acentuado & 5 \\
\hline Border collie/2012/F & 5 anos & Dificuldade de locomoção e dor & Sem contagem & 7 \\
\hline $\mathrm{SRD} / 2012 / \mathrm{F}$ & 7 anos & Claudicação & Sem contagem & $7 * * * * *$ \\
\hline Rottweiler/2012/F & 10 anos & Claudicação & Sem contagem & SI \\
\hline Samoieda/2012/F & 8 anos & Dificuldade de locomoção & Sem contagem & SI \\
\hline $\mathrm{SRD} / 2013 / \mathrm{F}$ & 3 anos & Dificuldade de locomoção e andar rígido & Acentuado & $4 * * * * * *$ \\
\hline Maltês/2013/F & 4 anos & Dificuldade de locomoção e dor cervical & Moderado & 5 \\
\hline Poodle/2013/M & 9 anos & Dor cervical & Leve & 7 \\
\hline $\mathrm{SRD} / 2013 / \mathrm{F}$ & 12 anos & $\begin{array}{l}\text { Dificuldade de locomoção e aumento de } \\
\text { volume das articulações }\end{array}$ & Leve & 15 \\
\hline Dachshund/2013/M & 4 anos & Dificuldade de locomoção e andar rígido & Leve & 3 \\
\hline
\end{tabular}

M: macho; F: fêmea; SRD: Sem raça definida; SI: Sem Informação * Recidiva e óbito após um mês; **SM: Sem melhora/eutanásia após cinco meses; ***Recidiva após nove meses/eutanásia; **** Recidiva após oito meses e óbito; ***** Recidiva e óbito após um mês; ******Em tratamento. 
48kg), demonstrando que a doença pode acometer animais de pesos variados.

O principal sinal clínico observado foi dificuldade na locomoção (19 de 27 cães, 70\%), caracterizada pela dificuldade de subir e descer degraus, fraqueza ou quedas, em quatro cães como único achado, seguido de artralgia (48\%), claudicação (37\%), 40\% em um único membro e $60 \%$ em membros variados, andar rígido (22,2\%) e tumefação articular $(11,1 \%)$. No trabalho de JAQUES et al. (2002) $(n=40)$, dificuldade de locomoção, dor articular e claudicação também foram os achados mais frequentes.

A poliartrite pode induzir o clínico em suspeitar de uma doença neurológica, principalmente por desencadear fraqueza (paresia) nos pacientes, que culminava em quedas dos membros envolvidos. Para GOLDSTEIN (2010), uma maneira de diferenciar seria ausência de ataxia proprioceptiva durante avaliação da locomoção, procedimento realizado em todos os cães desta pesquisa.

Sinais sistêmicos, como febre, inapetência, fraqueza, anorexia e perda de peso, podem ser os únicos sinais clínicos observados em cães com poliartrite (HOPPER, 1993; GOLDSTEIN, 2010). Neste estudo, no entanto, todos os sinais sistêmicos estavam acompanhados de algum sinal clínico relacionado à articulação. Febre e inapetência foram as alterações sistêmicas mais comuns, sendo observada em cinco $(18,5 \%)$ e quatro $(14,8 \%)$ dos 27 cães avaliados, respectivamente. Já no estudo de JACQUES et al. (2002), 10\% dos cães apresentaram apenas sinais sistêmicos. De acordo com esses achados, sugere-se incluir a poliartrite no diagnóstico diferencial de cães com sinais sistêmicos de origem desconhecida.

Análise de líquido sinovial foi realizada em todos os cães. Em dois dos 27 cães $(7,4 \%)$, a artrocentese foi realizada em duas articulações, $4 / 27(14,8 \%)$ em três articulações, $17 / 27(63 \%)$ em quatro articulações e 4/27 (14,8\%) em mais de quatro articulações. No total, 104 amostras de fluido sinovial foram analisadas entre os 27 cães e, assim como no estudo de JACQUES et al. (2002), o carpo e joelho foram as articulações mais avaliadas.

Análise das características físicas do líquido sinovial inclui exame visual da cor, turbidez, viscosidade e volume, sendo consideradas normais no cão e gato quando estivessem com as seguintes características: claro, incolor, viscoso, livre de detritos e não coagular. $\mathrm{O}$ volume obtido está diretamente relacionado com o tamanho do cão, da articulação afetada e presença ou ausência de derrame articular (BOON, 1997; PARRY, 1999). Neste estudo, o exame físico da amostra foi avaliado através da coloração do líquido sinovial, sendo incolor em três cães, ambar em um cão, róseo em três cães e vermelho em quatro cães. A concentração de proteína total no líquido sinovial foi quantificada nas amostras através de refratometria em 18 cães (60 articulações), cujo valor médio foi de $4,16 \mathrm{~g} \mathrm{dL}^{-1}\left(1,5-3,0 \mathrm{~g} \mathrm{dL}^{-1}\right)$. Embora os resultados por este método não sejam indicadores reais do teor de proteína total, MACWILLIAMS \& FRIEDRICHS (2003) mencionaram que o valor da proteína total tende a aumentar com a inflamação e a exsudação de proteínas do plasma para o interior da articulação.

A inflamação da articulação mediante análise da contagem celular do líquido sinovial foi classificada como leve em quatro cães (18\%), moderada em um cão $(4,5 \%)$ e acentuada em 17 cães (77,5\%). Em cinco cães, a contagem total de células nucleadas do líquido sinovial não foi fornecida pelo laboratório, entretanto foi estimado o predomínio de células mononucleares em quatro cães e neutrófilos em um cão. No estudo de JAQUES et al. (2002), houve uma maior prevalência $(42,5 \%)$ de inflamações sinoviais moderadas (com contagem de leucócitos entre 10 e 20 células) e apenas $27,5 \%$ dos cães com resultados acima de 50 células contra $77,5 \%$ do presente trabalho.

A contagem total de leucócitos e a contagem diferencial dessas células são essenciais na análise e classificação do líquido sinovial (BOON, 1997; GIBSON et al., 1999). Os neutrófilos, quando presentes em líquidos sinoviais normais, são, em pequeno número, em média $5 \%$ a $12 \%$ do total de células nucleadas (BOON, 1997), as demais células são compostas por mononucleares, incluindo linfócitos e sinoviócitos. Assim como em outros estudos (JACQUES et al., 2002; CLEMENTS et al., 2004), o neutrófilo foi a célula mais encontrada no líquido sinovial dos cães deste estudo (14 cães, [52\%]), enquanto que as células mononucleares (macrófagos e linfócitos) predominaram em 13 casos (48\%).

Oito de 27 cães $(29,6 \%)$ apresentaram anemia não regenerativa, sete $(26 \%)$ tinham leucocitose, cinco trombocitopenia $(18,5 \%)$ e, em três cães, foi observada leucopenia (11\%). Os resultados da bioquímica sérica foram considerados normais em sete de 27 cães (26\%). As demais alterações encontradas incluíram hiperproteinemia (15 cães [55,5\%]), hipoalbuminemia (10 cães [37\%]), aumento da fosfatase alcalina (quatro cães $[14,8 \%]$ ) e hipocalcemia (um cão [3,7\%]). Estes achados corroboram os estudos de JACQUES et al. (2002), 
CLEMENTS et al. (2004) e RONDEAU et al. (2005) ao mencionarem que: 1) as alterações hematológicas ocorrem provavelmente devido à inflamação crônica, 2) a elevação da fosfatase alcalina provavelmente é secundária à liberação de glicocorticoides endógenos e 3) a hipoalbuminemia é secundária à resposta de fase aguda inflamatória. Hiperproteinemia, provavelmente secundária à resposta inflamatória de fase aguda, foi um achado comum nos casos aqui descritos.

$\mathrm{O}$ exame radiográfico foi realizado em 20 dos 27 cães para distinguir poliartrite erosiva da não erosiva. As articulações submetidas à radiografia incluíram joelho (16 cães), coxofemoral (7), carpo (6), cotovelo (2) e coluna vertebral (8). Desses vinte cães, seis apresentavam alterações radiográficas. Quatro cães $(20 \%)$ apresentavam evidência radiográfica de degeneração articular, um desses também apresentava osteopenia, um (5\%) tinha evidência de derrame articular e um $(5 \%)$ demonstrou presença de osteófitos nos carpos.

As radiografias da coluna vertebral foram realizadas em $8 / 27$ (29,6\%), sendo um da região cervical, uma toracolombar, quatro lombossacras e duas nas regiões cervical e toracolombar. Foram normais em $6 / 8$ dos exames $(87,5 \%)$. Os cães com alterações radiográficas na coluna vertebral apresentavam anormalidades degenerativas, caracterizado por proliferação óssea na extremidade cranioventral da vértebra sacral e outro por osteófitos vertebrais.

A característica radiográfica mais importante da artrite erosiva é a presença de erosões envolvendo a superfície articular (GOLDSTEIN, 2010). As lesões são progressivas, caracterizadas por alterações no espaço articular ou na forma de focos radioluscentes de vários tamanhos (GOLDSTEIN, 2010) e deformidades articulares, incluindo luxações e subluxações (CARR \& MICHELS, 1997). Com base nestes critérios, PA erosiva não foi identificado em nenhum dos cães deste estudo, sugerindo que a PA não erosiva é a mais frequente. Em outro estudo de PA (sépticas e não sépticas) em cães, alterações erosivas foram observadas em $3 \%$ dos casos que tinham radiografias das articulações (RONDEAU et al., 2005).

Para JACQUES et al. (2002), RONDEAU et al. (2005) e STULL et al. (2008), as PA comumente relatadas são imunomediadas (idiopática), entretanto, podem ter uma falsa representação, devido à dificuldade em estabelecer a etiologia. No presente estudo, não foi possível classificar as PA quanto à etiologia, devido a não realização dos exames complementares necessários para exclusão dos diagnósticos diferenciais. Processos inflamatórios ou infecciosos locais ou distantes das articulações, doenças hepáticas crônicas, gastrointestinais, neoplasias, reações vacinais ou induzidas por drogas devem ser investigadas como possíveis causas (BENNETT, 2010). A melhora clínica dos pacientes em resposta ao tratamento com drogas imunossupressoras reforçou a suspeita de PA idiopática.

Ofármacomaisutilizado para otratamento de cães com PA é o corticosteroide (CLEMENTS et al., 2004, JOHNSON \& MACKIN, 2012b). No estudo de CLEMENTS et al. (2004), 33\% dos cães tratados com prednisolona apresentaram melhora clínica, já nos cães desta pesquisa, 18 cães foram submetidos ao tratamento, sendo que em dezesseis $(89 \%)$ observou-se melhora dos sinais. Em um cão $(5,5 \%)$, foi associada ciclofosfamida (dose $50 \mathrm{mg} \mathrm{m}^{-2}$, via oral, a cada 24 horas, durante duas semanas) e em outro azatioprina ( $2 \mathrm{mg} \mathrm{kg}^{-1}$, via oral, a cada 24 horas, durante 7 dias, após, a cada 48 horas, durante duas semanas), ambos apresentaram melhora clínica e citológica após dois meses e outro ainda está em tratamento, respectivamente. Nove cães (33\%) não retornaram para reavaliação, portanto, não foi possível obter informações sobre a evolução desses casos. Com base nos resultados da terapia, podemos inferir que pelo menos 16 casos são imunomediados.

O tratamento é realizado até que não haja evidência citológica de inflamação e os sinais clínicos não estejam mais presentes (dor, claudicação, edema, dificuldade de locomoção, etc.) (JOHNSON \& MACKIN, 2012b). Falha no tratamento pode resultar em persistência ou recorrência desses sinais (COLOPY et al., 2010). Outros fármacos como quimioterápicos e imunomoduladores são alternativas eficazes para o tratamento de cães com poliartrite (CLEMENTS et al., 2004; COLOPY et al., 2010; JOHNSON \& MACKIN, 2012b).

Segundo CLEMENTS et al. (2004), 56\% $(n=39)$ dos cães tratados apresentaram remissão total dos sinais e $15 \%$ foram submetidos à eutanásia ou morreram pela progressão da doença, resultado semelhante ao encontrado nesta pesquisa, ou seja, (67\% [12/18] e $28 \%$ [5/18], respectivamente). Neste estudo, dos 18 cães submetidos ao tratamento, 44\% (8/18) apresentaram melhora dos sinais clínicos na primeira semana, $39 \%(7 / 18)$ no primeiro mês, $5,5 \%(1 / 18)$ no $38^{\circ}$ dia, $5,5 \%(1 / 18)$ não apresentaram melhora durante cinco meses de tratamento e $5,5 \%(1 / 18)$ estão em tratamento. Esses resultados demonstraram uma variação 
individual na resposta terapêutica com o mesmo protocolo terapêutico na maioria dos casos. Por outro lado, o grau de inflamação possivelmente não influenciou no tempo de recuperação, conforme demonstrado na tabela 1 .

Quanto à recidiva dos sinais clínicos, dos 18 cães tratados, em quatro (22,2\%), ocorreram dentro de nove meses, sendo que três foram a óbito, não sendo possível determinar a causa e sua relação com a poliartrite. Um cão $(5,5 \%)$ foi submetido à eutanásia por solicitação do proprietário e a necropsia revelou artrite supurativa acentuada em várias articulações, sugerindo poliartrite erosiva e que provavelmente interferiu na recuperação do paciente.

\section{CONCLUSÃO}

Por meio dos estudos realizados nos registros médicos do Serviço de Neurologia do HVU-UFSM, pode-se concluir que a poliartrite é uma doença relativamente comum e acomete cães de diferentes raças e idades, cujos sinais clínicos mais frequentes são dificuldade de locomoção, dor articular e claudicação. A prednisolona em doses imunossupressoras é efetiva no controle da afecção na maioria dos casos e a remissão somente dos sinais clínicos não determina a cura da doença e nem a interrupção do tratamento.

\section{REFERÊNCIAS}

BENNETT, D. Immune-mediated and infectious arthritis. In: ETTINGER, J.E.; FELDMAN, E.C. (Eds,). Textbook of veterinary internal medicine. 5.ed. Philadelphia: Saunders, 2010, p.1958-1965.

BOON, D. Synovial fluid analysis: a guide for small-animal practitioners. Veterinary Medicine, v.92, n.5, p.443-451, 1997.

CARR, A.P.; MICHELS, G. Identifying noninfectious erosive arthritis in dogs and cats. Veterinary Medicine, v.92, n.9, p.804$810,1997$.

CLEMENTS, D.N. et al. Type I immune-mediated polyarthritis in dogs: 39 cases (1997-2002). Journal of the American Veterinary Medical Association, v.224, n.8, p.1323-1327, 2004. Disponível em: <http://www.ncbi.nlm.nih.gov/pubmed/15112783>. Acesso em: 21 ago. 2013. doi: 10.2460/javma.2004.224.1323.

COLOPY, S.A. et al. Efficacy of leflunomide for treatment of immune-mediated polyarthritis in dogs: 14 cases (2006-2008). Journal of the American Veterinary Medical Association, v.236, n.3, p.312-318, 2010. Disponível em: <http://www.ncbi. nlm.nih.gov/pmc/articles/PMC3089001/pdf/nihms286325.pdf>. Acesso em: 10 nov. 2012. doi:10.2460/javma.236.3.312.

DE HAAN, J.J.; ANDREASEN, C.B. Calcium crystalassociated arthropathy (pseudogout) in a dog. Journal of the
American Veterinary Medical Association, v.200, n.7, p.943946, 1992.

GIBSON, N.R. et al. Value of direct smears of synovial fluid in the diagnosis of canine joint disease. Veterinary Record, v.144, n.17, p.463-465, 1999. Disponível em: <http://www.ncbi.nlm.nih. gov/pubmed/10358874>. Acesso em: 22 ago. 2013. doi:10.1136/ vr.144.17.463.

GOLDSTEIN, R.E. Swollen joints and lameness. In: ETTINGER, J.E.; FELDMAN, E.C. (Eds.). Textbook of veterinary internal medicine. 5.ed. Philadelphia: Saunders, 2010. p.83-87.

HOPPER, P.E. Immune-mediated joint diseases. In: SLATTER, D. (Ed.). Textbook of small animal surgery. 2.ed. Philadelphia: Saunders, 1993. p.1928-1937.

JACQUES, D. et al. A retrospective study of 40 dogs with polyarthritis. Veterinary Surgery, v.31, n.5, p.428-434, 2002. Disponível em: <http://cmapspublic3. ihmc.us/rid\%3D 1223226458328 _466978485_43604/ Polyarthritis\%2520retrospective.pdf>. Acesso em: 19 de nov. 2012. doi: $10.1053 /$ jvet.2002.34665.

JOHNSON, K.C.; MACKIN, A. Canine immune-mediated polyarthritis. Part 1: pathophysiology. Journal of the American Animal Hospital Association, v.48, n.1, p.12-17, 2012a. Disponível em: <http://www.jaaha.org/content/48/1/12.abstract $>$. Acesso em: 10 jul. 2013. doi: 10.5326/JAAHA-MS-5744.

JOHNSON, K.C.; MACKIN, A. Canine immune-mediated polyarthritis. Part 2: diagnosis and treatment. Journal of the American Animal Hospital Association, v.48, n.2, p.71-82, 2012b. Disponível em: <http://www.jaaha.org/content/48/1/12. abstract $>$. Acesso em: 10 jul. 2013. doi:10.5326/JAAHAMS-5756.

MACWILLIAMS, P.S.; FRIEDRICHS, K.R. Laboratory evaluation and interpretation of synovial fluid. Veterinary Clinics of North America: Small Animal Practice. v.33, n.1, p153-178, 2003.

MCCONKEY, S.E. et al. Leishmanial polyarthritis in a dog. Canadian Veterinary Journal, v.43, n.8, p.607-609, 2002. Disponível em: <http://www.ncbi.nlm.nih.gov/pmc/articles/ PMC339396/pdf/20020800s00025p607.pdf>. Acesso em: 09 out. 2012.

PARRY, B.W. Synovial fluid. In: COWELL, R.L. et al. (Eds.). Diagnostic cytology and hematology of the dog and cat. 2.ed. St Louis: Mosby, 1999. p.104-119.

RONDEAU, M.P. et al. Suppurative, nonseptic polyarthropathy in dogs. Journal of Veterinary Internal Medicine, v.19, n.5, p.654-662, 2005. Disponível em: <http://onlinelibrary.wiley.com/ doi/10.1111/j.1939-1676.2005.tb02743.x/pdf. $>$. Acesso em: 22 out. 2012.

SANTOS, M. et al. Polyarthritis associated with visceral leishmaniasis in a juvenile dog. Veterinary Parasitology, v.141, n.3-4, p.340-344, 2006. Disponível em: <http://www.sciencedirect. com/science/article/pii/S0304401706003451\#>. Acesso em: 21 nov. 2012

SPRENG, D. Leishmanial polyarthritis in two dogs. Journal of Small Animal Practice, v.34, n.11, p.559- 
563, 1993. Disponível em: <http://onlinelibrary.wiley.com/ doi/10.1111/j.1748-5827.1993.tb03548.x/abstract>. Acesso em: 12 jul. 2013. doi:10.1111/j.1748-5827.1993.tb03548.x.

STULL, J.W. et al. Canine immune-mediated polyarthritis: clinical and laboratory findings in 83 cases in western Canada (1991-2001). Canadian Veterinary Journal, v.49, n.12, p.11951203, 2008. Disponível em: <http://www.ncbi.nlm.nih.gov/pmc/ articles/PMC2583415/>. Acesso em: 21 nov. 2012.

THILAGAR, S. et al. An unusual case of ehrlichiosis associated with polyarthritis in a dog - A case report. Indian Veterinary Journal, v.67, n.3, p.267-268, 1990.

THOREN-TOLLING, K.; RYDEN, L. Serum autoantibodies and clinical/pathological features in German sheperd dogs with a lupus like syndrome. Acta Veterinary Scandinavica, v.32, n.1, p.15-26, 1991.
WEBB, A.A. et al. Steroid-responsive meningitis-arteritis in dogs with noninfectious, nonerosive, idiopathic, immune-mediated polyarthritis. Journal of Veterinary Internal Medicine, v.16, n.3, p.269-273, 2002. Disponível em: <http://onlinelibrary.wiley.com/ doi/10.1111/j.1939-1676.2002.tb02368.x/pdf>. Acesso em: $12 \mathrm{dez}$. 2012.

WILSON-WAMBOLDT, J. Type I idiopathic non-erosive immune-mediated polyarthritis in a mixed-breed dog. Canadian Veterinary Journal, v.52, n.2, p.192-196. 2011. Disponível em: $<$ http://www.ncbi.nlm.nih.gov/pmc/articles/PMC3022464/pdf/ cvj_02_192.pdf $>$. Acesso em: 13 nov. 2012.

WOODARD, J.C. et al. Erosive polyarthritis in two greyhounds. Journal of the American Animal Hospital Association, v.198, n.5, p.873-876, 1991. 